\title{
Seek and Decode: Random Multiple Access with Multiuser Detection and Physical-Layer Network Coding
}

\author{
Giuseppe $\mathrm{Cocco}^{\dagger}$, Stephan Pfletschinger* \\ ${ }^{\dagger}$ German Aerospace Center - DLR \\ Oberpfaffenhofen, D-82234, Wessling, Germany \\ * Centre Tecnològic de Telecomunicacions de Catalunya - CTTC \\ Parc Mediterrani de la Tecnologia, Av. Carl Friedrich Gauss 7 08860, Castelldefels - Spain \\ gcocco81@gmail.com, stephan.pfletschinger@cttc.es
}

\begin{abstract}
We present a novel random multiple access scheme that combines joint multiuser detection (MUD) with physicallayer network coding (PLNC) over extended Galois fields (EGF). We derive an analytical bound to the throughput at the system level and present simulation results for the decoding at the physical level in both fast fading and block fading channels. We adopt a cross layer approach in which a non-binary joint multiuser decoder is used in combination with PLNC at slot level, while the use of EGF increases the system diversity at frame level. The results we present are encouraging and suggest that the combination of these two interference management techniques can significantly enhance the performance of random multiple access systems.
\end{abstract}

\section{INTRODUCTION}

Random access systems (RAS) are at the same time an opportunity and a challenge. Opportunity because they do not require (or require little) coordination among the transmitters, which, among other advantages, makes it possible to live together with large delays, as in like satellite communication networks. However, if on the one hand the lack of coordination can be seen as an asset, on the other hand it brings about the issue of signals from differen transmitters interfering at the receiver. The problem of collisions in RAS has been addressed in different ways such as the exploitation of the difference in the power of the received signals [1] and the application of multiuser detection (MUD) methods as in the code-division multiple access (CDMA) systems [2]. Multipacket reception, i.e., the capability for the receiver to decode more than one packet from a collision, has been and still is an active research field. In [3], an overview of the main multiuser detection techniques is presented. The impact of multi-packets reception capability in slotted ALOHA systems has been studied in [4]. Another approach proposed in the literature consists in having each transmitter sending multiple replicas of the same packet within a frame. The receiver tries to decode the packets that do not experience collision [5] or subtracts the decoded packets from the slots where their replicas are [6][7]. The scheme proposed in [6] has been enhanced in [8] by inducing fluctuations in the received power in order to allow iterative hard interference cancelation within single slots. Recently the possibility of decoding functions of colliding signals has been studied from an information theoretical point of view and assuming lattice codes [9][10]. In [11] the linearity of error correction codes has been applied for the decoding of the bitwise XOR of the colliding signals in the two-way relay channel (TWRC) under the assumption of equal codes at both end nodes. This approach is one of the possible implementations of the wider concept known as physical-layer network coding (PLNC). Most part of the literature on PLNC focuses on the TWRC. In [12] a generalized sum-product algorithm has been proposed for PLNC in the MAC phase of the TWRC. In [13] a quaternary decoding approach for the MAC phase of the two-way relay channel has been proposed, showing that there is an advantage in obtaining the bitwise sum by combining the previously estimated individual messages rather than directly decoding the sum from the analog signal. In [14] it has been proposed to apply PLNC in slotted random multiple access systems by decoding the bitwise XOR of all colliding signals within a slot and then trying to recover all transmitted packets within a frame using matrix manipulations in $G F(2)$. In [15] and [16] an enhanced scheme based on PLNC over extended Galois fields has been proposed, showing an increased system diversity.

In the present paper we propose a random multiple access scheme for symbol-synchronous slotted ALOHA systems named Seek and Decode (S\&D) in which the transmitters pre-encode their information messages multiplying them by a random coefficient in an extended Galois field while the receiver tries to decode any linear combination in $G F(2)$ from the set of colliding bursts within each slot. The decoding process is an hybrid between a joint multiuser decoder and a PLNC decoder. Once the whole frame has been processed at the physical layer, the receiver uses the whole set of linear combinations available to retrieve all messages transmitted within the frame by using matrix manipulation techniques over the extended Galois field used in the pre-coding stage. The use of an extended Galois field in the pre-coding stage 
increases system diversity. We derive an upper bound to the throughput at the system level and present numerical results for the number of innovative messages decoded within a slot in a block fading channel. FER curves for the fast fading channel are also presented.

\section{System MOdeL}

Let us consider a random multiple access network with a population of $M$ transmitting terminals $\mathrm{T}_{1}, \ldots, \mathrm{T}_{M}$, and one receiver $R$. In the rest of the paper we will use interchangeably the terms "transmitting node", "terminal node" and "transmitter". Time is divided into slots. We define a packet $\mathbf{u}$ as a block of $R N$ information bits. Each terminal generates packets according to a Poisson process of intensity $\frac{G}{M}$ packets per slot. The overall load offered to the network is, thus, $G$ packets per slot. Each time a packet $\mathbf{u}_{i}=\left[u_{i, 1}, \ldots, u_{i, R N}\right]$ is generated at terminal $\mathrm{T}_{i}$, it is channel encoded using an encoder of rate $R$ creating a codeword $\mathbf{c}_{i}=\left[c_{i, 1}, \ldots, c_{i, N}\right]$ of $N$ symbols. The same channel code is used by all transmitting nodes. The codeword $\mathbf{c}_{i}$ is then mapped to a binary phase-shift keying (BPSK)-modulated burst $\mathbf{x}_{i}$ and transmitted over the channel. We consider BPSK modulation for simplicity, but other kinds of modulations can also be used. We assume that the burst duration is approximately equal to that of a slot. Transmissions are organized in frames of $S$ slots each. We further assume that the transmitters are synchronized such that all signals transmitted within a slot add up with symbol synchronism at the receiver. At the receiver side, $\mathrm{R}$ tries to decode as many linearly independent messages as possible by applying both joint multi-user detection and physical layer network coding. In order to increase system diversity at the frame level, we assume that a pre-coding, such as the one in [16], is applied by each of the terminals before the channel encoding. The main innovation in the present work is in the type of decoder used and in the fact that the receiver tries to obtain all possible linear combinations in $G F(2)$ from the signals colliding within a slot. Finally, such linear combinations are then used by the receiver to recover the whole frame, treating the set of decoded linear combinations across the whole frame as a system of equations in $G F\left(2^{n}\right)$.

\section{RANDOM ACCESS WITH MUD AND PLNC}

In the present section we describe the proposed random access scheme named Seek and Decode (S\&D). The transmitter side is the same as in [16]. The main innovation is in the decoding process at both slot level and frame level. The receiver processes one slot at a time in the analog domain trying to decode either single messages or some linear combination of the colliding signals. We briefly recall the operations at the transmitter side presented in [16] and then move to the description of the receiver side.

\section{A. Transmitter Side}

Each message is transmitted more than once within a frame, i.e., several replicas of the same message (bursts) are transmitted. Assume that node $i$ has a message $\mathbf{u}_{i}$ to deliver to $\mathrm{R}$ during a given frame, i.e., node $\mathrm{T}_{i}$ is an active terminal. Before each transmission, node $i$ pre-encodes $\mathbf{u}_{i}$ as depicted in Fig. 1. The message to be transmitted is divided into sub-blocks.

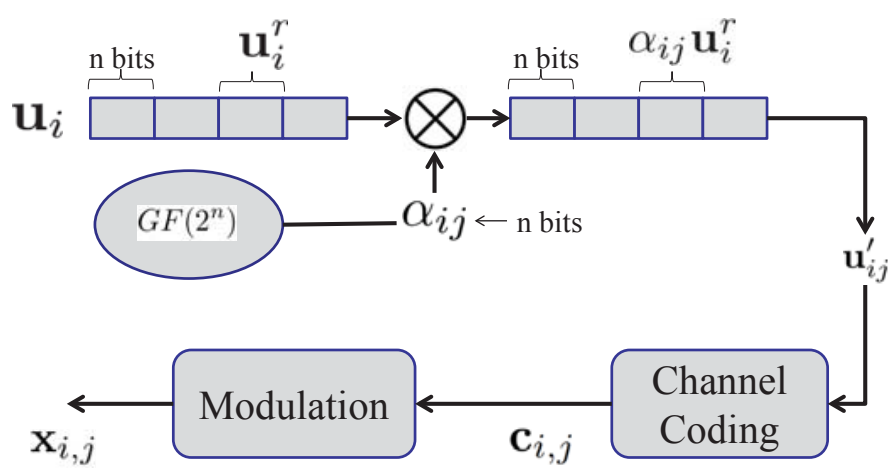

Fig. 1. Pre-coding, channel coding and modulation scheme at the transmitter side. Pre-coding consists in dividing the message into blocks of $n$ bits (indicated as $\mathbf{u}_{i}^{r}$ in the figure) and multiply each of these blocks by the same coefficient randomly chosen in $G F\left(2^{n}\right)$. The sub index $j$ indicates the slot within a frame in which the replica of message $\mathbf{u}_{i}$ is transmitted. A different coefficient $\alpha_{i j}$ is used for each replica.

Each sub-block is multiplied by a coefficient $\alpha_{i j} \in G F\left(2^{n}\right)$. Coefficient $\alpha_{i j}, j \in\{1, \ldots, S\}$ is chosen at random in each time slot $j$ while it is fixed for all sub-blocks within a message. Note that the pre-coding does not have any impact on the decoding process at the physical layer. The multiplication of $\mathbf{u}_{i}$ by $\alpha_{i j}$ is needed to increase diversity at the frame level and does not modify the number of information bits transmitted. After the multiplication, the message is channel-encoded, a header is attached and the modulation takes place. The header can be generated using a pseudonoise sequence generator such as the ones used in CDMA. In practice the coefficients $\alpha_{i j}$ can be generated using a pseudo-random number generator. In a given frame the active node chooses a different seed and uses as many outputs of the generator as the number of replicas transmitted within the frame. Each seed is associated to a certain header, which is assumed to be detected by the receiver using the cross-correlation properties of the header ${ }^{1}$. The same header is used within a given frame by an active node. In this way the receiver can detect in which slots a certain node is transmitting and derive the coefficients used in the different replicas from the header. The header is also used to perform the channel estimation of each of the transmitters. A more detailed analysis of the issues related to header detection and channel estimation can be found in [16] and [17].

\section{B. Receiver Side}

The main innovation of the proposed random access scheme with respect to previous works is at the receiver side. In literature, and up to our knowledge, when a receiver receives more than one interfering signal, it can either use some kind of interference cancelation or, as in physical layer network coding, try to decode a function of the colliding messages.

\footnotetext{
${ }^{1}$ Note that other signatures can also be used by the nodes to allow $R$ for the identification of the transmitters.
} 
Most of the multiuser detection techniques found in literature can be categorized as parallel (PIC) or serial (SIC). Often such methods are iterative and alternate a detection phase to an estimation phase. In the proposed scheme the receiver applies a joint decoder which tries to recover simultaneously all messages involved in the collision. An FFT-based belief propagation decoder over the vectorial combination of all message bits, which is described in detail in the companion paper [18], has been adopted. The decoder jointly estimates all the single messages and then calculates the bitwise XOR of any subset of the estimated messages. It is important to notice that, as shown in [13], the sum in $G F(2)$ of a set of estimated messages can be correct even if the estimated messages taken individually contain errors. A cyclic redundancy check (CRC) can be used for error detection. Note that, due to the linearity of the code, the XOR of the CRCs relative to a set of messages is a valid CRC for the XOR of the messages in the set. Ideal error detection at the receiver is assumed in the following for ease of exposition.

Given a slot with a collision of size $k$, the receiver tries to decode $k$ independent linear combinations in $G F(2)$ of the colliding signals. One possible way to proceed, although not necessarily the optimal one, can be the following. For each slot with a collision of size $k$, the decoder tries to decode single messages. If less than $k$ messages are decoded correctly, the decoder tries to decode the sum in $G F(2)$ of pairs of messages (there are $\left(\begin{array}{l}k \\ 2\end{array}\right)$ possible combinations) stopping when a total of $k$ linearly independent combinations are decoded. If still less than $k$ linear combinations have been decoded, sums of three messages are considered. The process goes on until either enough linear combinations have been decoded or all possible combinations have been exhausted. The total number of linear combinations that the decoder can try to recover is $\sum_{i=1}^{k}\left(\begin{array}{c}k \\ i\end{array}\right)=2^{k}-1$.

\section{Example}

In the following we illustrate the S\&D scheme with a toy example. Let us consider a frame with $S=2$ slots and four active nodes. Let us assume that nodes 1 and 2 transmit in both slots, each time choosing at random their pre-coding coefficients. Node 3 only transmits in the first slot while node 4 transmits only in the second, as illustrated in Fig. 2. Let us assume that the S\&D decoder is able to output only two linear combinations in each of the two slots as shown in the picture. The receiver tries, then, to recover all information messages $\mathbf{u}_{1} \ldots, \mathbf{u}_{4}$. The decoding is possible if the coefficient matrix A in $G F\left(2^{n}\right)$ (shown below) has full rank.

$$
\mathbf{A}=\left(\begin{array}{cccc}
0 & 0 & \alpha_{2,1} & \alpha_{1,1} \\
0 & \alpha_{3,1} & 0 & \alpha_{1,1} \\
0 & 0 & \alpha_{2,2} & \alpha_{1,2} \\
\alpha_{4,2} & 0 & \alpha_{2,2} & 0
\end{array}\right)
$$

Note that matrix $\mathbf{A}$ is rank deficient if coefficients are chosen in $G F(2)$ (i.e., all coefficients shown in the matrix above are equal to 1 ), while it can be full rank in some extended Galois

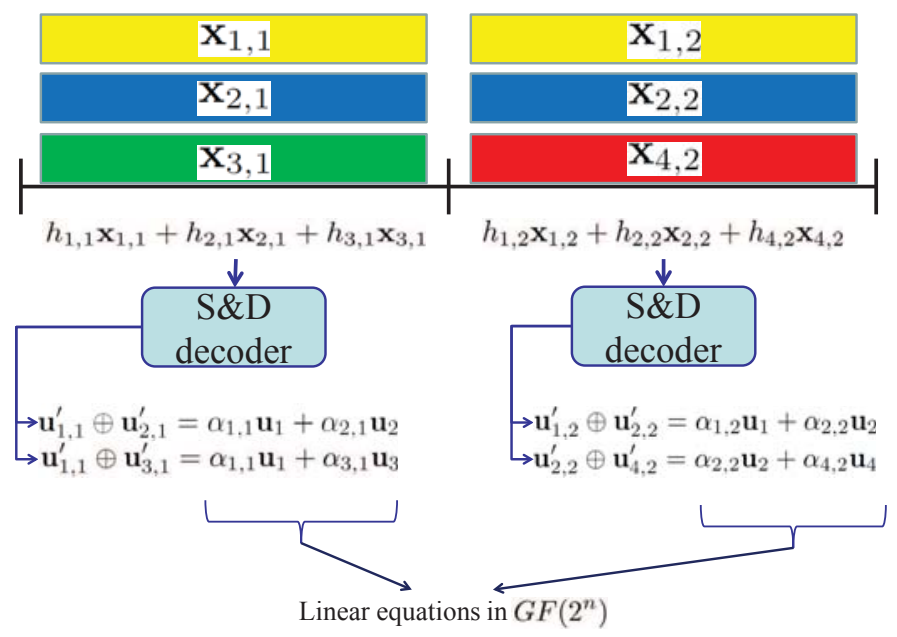

Fig. 2. Example of decoding at the physical layer in S\&D with a two-slots frame and four active terminals. Nodes 1 and 2 transmit in both slots, each time choosing at random their pre-coding coefficients. Node 3 only transmits in the first slot while node 4 transmits only in the second.

field. For instance, in $G F(4)$ the matrix

$$
\mathbf{A}=\left(\begin{array}{llll}
0 & 0 & 1 & 1 \\
0 & 2 & 0 & 1 \\
0 & 0 & 2 & 3 \\
1 & 0 & 2 & 0
\end{array}\right)
$$

is full rank. If, by choosing the coefficients at random, the above matrix is obtained it would be possible to decode the whole frame. The probability of obtaining a full rank matrix increases with the field size. Finally, we note that in the example the average number of packets decoded per slot is 2.

\section{Throughrut ANALYSIS}

In the present section we derive an upper bound to the system throughput, defined as the average number of decoded messages per time slot, for the proposed scheme. The throughput depends on the repetition strategy chosen. For mathematical tractability we assume a general scheme in which each active node transmits in each slot with probability $p$, fixed for all nodes.

\section{A. Upper Bound to Decoding Probability}

In our simulation results we observed that the probability of correct decoding for the sum of a subset of messages with cardinality $i$ from a collision of size $k, i \leq k$, is a function of both $i$ and $k$ in both fast fading and block fading channels. We define:

$\operatorname{Pr}\{$ decode sum of $i$ messages form collision of $k\} \triangleq p_{k, i}$.

$p_{k, i}$ can be upper bounded as follows:

$$
p_{k, i} \leq \ddot{p}_{k, i} \leq \tilde{p}_{k, i},
$$

where

$$
\ddot{p}_{k, i} \triangleq \max _{S_{k, i}} p_{k, i}
$$


$S_{k, i}$ being one of the $\left(\begin{array}{c}k \\ i\end{array}\right)$ subsets of $i$ messages among the $k$, while

$$
\tilde{p}_{k} \triangleq \max _{i} \ddot{p}_{k, i} \text {. }
$$

We found through simulations that $p_{k, i}$ is lower than or equal to the probability to decode the sum of the $i$ strongest signals among the $k$. Thus, $\tilde{p}_{k}$ is the maximum across all subset sizes $i, i \in\{1, \ldots, k\}$, of the probability to decode the sum of the $i$ strongest signals. $\tilde{p}_{k}$ is used in the following for the derivation of the upper bound to the throughput. By applying both joint MUD and PLNC, the receiver can obtain up to $\eta_{k} \triangleq 2^{k}-1$ different linear combinations in a slot with a collision of size $k$. At most $k$ of the decoded combinations are linearly independent. For ease of calculation we assume in the following that the decodability of a given combination is independent of the decoding of any other within the same slot ${ }^{2}$. The number of combinations (that can be linearly independent or not) decoded in a slot is a random variable. We indicate such variable with $\epsilon$. Let us now indicate with $\epsilon_{k}$ the number of combinations decoded in a slot when the collision size is $k$. $\epsilon_{k}$ is a Binomial random variable with parameters $\tilde{p}_{k, i}$ and $\eta_{k}$, i.e., $\epsilon_{k} \sim B\left(\eta_{k}, \tilde{p}_{k}\right)$. The mean and variance of $\epsilon_{k}$ are $\eta_{k} \tilde{p}_{k}$ and $\eta_{k} \tilde{p}_{k}\left(1-\tilde{p}_{k}\right)$, respectively. The mean value of $\epsilon E[\epsilon]=\bar{\epsilon}$ is then:

$$
\bar{\epsilon}=\sum_{k=1}^{N^{t x}}\left(\begin{array}{c}
N^{t x} \\
k
\end{array}\right) p^{k}(1-p)^{N^{t x}-k} \eta_{k} \tilde{p}_{k},
$$

while the mean squared value of $\epsilon$ is:

$$
\begin{aligned}
E\left[\epsilon^{2}\right]= & \sum_{k=1}^{N^{t x}}\left(\begin{array}{c}
N^{t x} \\
k
\end{array}\right) p^{k}(1-p)^{N^{t x}-k} \times \\
& \times\left[\eta_{k} \tilde{p}_{k}\left(1-\tilde{p}_{k}\right)+\left(\eta_{k} \tilde{p}_{k}\right)^{2}\right] .
\end{aligned}
$$

Finally, the variance of $\epsilon \sigma_{\epsilon}^{2}$ can be calculated using expressions (1) and (2) as $\sigma_{\epsilon}^{2}=E\left[\epsilon^{2}\right]-\bar{\epsilon}^{2}$. The total number of combinations decoded in the whole frame is a random variable given by the sum of the numbers of combinations decoded in all slots, which are i.i.d. random variables and for which we just calculated the mean and the variance. Practical values for $S$ can be on the order of 100, which is large enough to approximate the sum of $S$ i.i.d. random variables as a Gaussian variable having mean $S \bar{\epsilon}$ and variance $S \sigma_{\epsilon}$. From expressions (1) and (2) it can be seen that the mean and the variance of $\epsilon_{f r}$ depend on the number of active terminals in the frame. For this we indicate with $\bar{\epsilon}\left(N^{t x}\right)_{f r}$ and $\sigma\left(N^{t x}\right)_{\epsilon_{f r}}^{2}$ the mean and the variance of $\epsilon_{f r}$, respectively. As mentioned in Section II we assume Poisson arrivals with an overall offered load of $G$ packets per slot. An upper bound to the normalized throughput can be calculated by assuming $p=2^{n}-1, n$ being the size of the Galois field of the coefficients used in the pre-coding step, and assuming that all combinations decoded within a frame

\footnotetext{
${ }^{2}$ In general this is not strictly true, since giving the correct decoding of a subset of individual messages, any combination of such messages can also be decoded. However, it can happen that the single messages can not be decoded while the sum can (e.g., this is true for certain code rates and if two signals have the same channel amplitude as shown in [13].)
}

are obtained using independently drawn coefficients for each message in each equation ${ }^{3}$. If $n$ is large, the probability to decode a number of combinations larger than or equal to the number of active nodes within a frame is given by (3)

$$
\sum_{N^{t x}=1}^{\infty} \frac{(G S)^{N^{t x}} e^{-G S}}{N^{t x} !} \sum_{m=N^{t x}}^{S\left(2^{N^{t x}}-1\right)} \frac{e^{-\frac{\left(m-\bar{\epsilon}(m)_{f r}\right.}{\left.2 \sigma(m)_{\epsilon}\right)_{f r}^{2}}}}{\sqrt{2 \pi \sigma(m)_{\epsilon_{f r}}^{2}}}
$$

Using (3) we can obtain an upper bound to the normalized system throughput $\Phi_{U B}$ when a large field size is used. The expression for $\Phi_{U B}$ is given by Eqn. 4 at the top of next page.

\section{NUMERICAL RESUlts}

\section{A. Joint Decoding in the Fast Fading Channel}

For a first evaluation of the joint decoding approach, we considered the simultaneous decoding of $k \in\{2,3, \ldots, 8\}$ packets in a fast Rayleigh fading symmetric multiple-access channel, given by

$$
y_{n}=\sum_{i=1}^{k} h_{i, n} \cdot x_{i, n}+w_{n}, w_{n} \sim \mathcal{N}(0,1) .
$$

The fading coefficients $h_{i, n}$ are i.i.d. Rayleigh distributed with average signal-to-noise ratio $\mathrm{SNR}=E\left[h_{i, n}^{2}\right]$ and the decoder employs joint decoding of all $k$ messages as described in [18]. Due to the fast fading and the symmetric channel, the $k$ transmitted packets experience approximately the same channel quality and we consider the word error rate of the combined messages. In other words, we count a word error if any of the $k$ messages is not correctly decoded. Fig. 3 shows the simulation results for the CCSDS LDPC code [19] of rate $R=0.4$ and message length $R N=1024$ bits. In Fig. 3 we can see that the simultaneous decoding of several packets is possible and requires only a moderate SNR increase for a growing collision size.

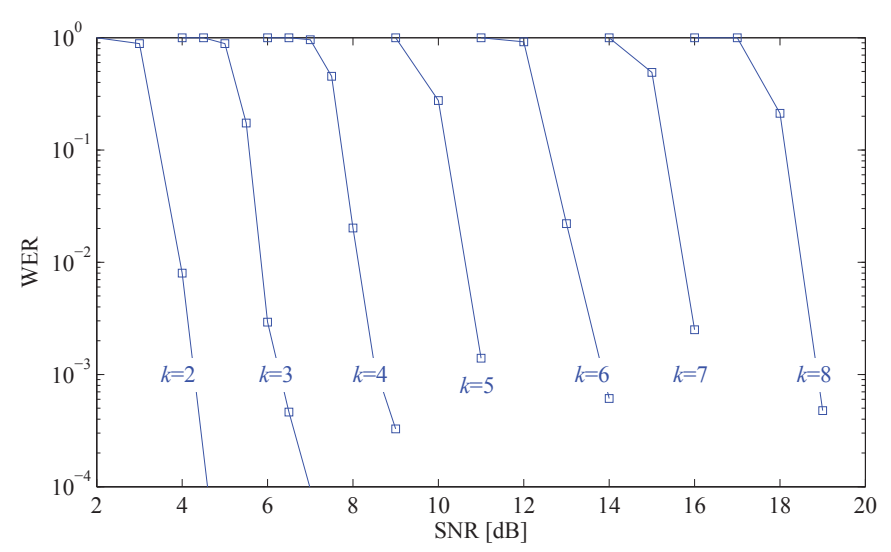

Fig. 3. Word error rate for joint decoding in symmetric fast Rayleigh fading channel. An error occurs when one of the $k$ messages can not be correctly decoded.

\footnotetext{
${ }^{3}$ Note that in practice each message has the same coefficient for all
} combinations within a given slot. 


$$
\Phi_{U B}=\frac{1}{S} \sum_{N^{t x}=1}^{\infty} N^{t x} \frac{(G S)^{N^{t x}} e^{-G S}}{N^{t x} !} \sum_{m=N^{t x}}^{S\left(2^{N^{t x}}-1\right)} \frac{e^{-\frac{\left(m-\bar{\epsilon}(m)_{f r}\right)^{2}}{2 \sigma(m))_{f r}^{2}}}}{\sqrt{2 \pi \sigma(m)_{\epsilon_{f r}}^{2}}}=G \sum_{N^{t x}=0}^{\infty} \frac{(G S)^{N^{t x}} e^{-G S}}{N^{t x} !} \sum_{m=N^{t x}}^{S\left(2^{N^{t x}}-1\right)} \frac{e^{-\frac{\left(m-\bar{\epsilon}(m)_{f r}\right)^{2}}{2 \sigma(m)_{\epsilon}^{2}}}}{\sqrt{2 \pi \sigma(m)_{\epsilon_{f r}}^{2}}} .
$$

While these results verify the functioning of the joint decoding approach, for a practical random access scheme the assumption of fast fading is not realistic and we therefore apply block fading for the following throughput evaluations.

\section{B. Seek \& Decode in the Block Fading Channel}

As described in the previous sections, in the $S \& D$ scheme the receiver uses a joint decoder to recover as many linearly independent combinations of colliding signal as possible in each slot and then, exploiting the pre-coding of the transmitted messages at the receiver side, try to decode the whole frame using standard matrix manipulations techniques. The linearly independent combinations may be either single messages or sums in $G F(2)$ of any number of messages from 2 to $k, k$ being the collision size in the slot. One could guess that it might not be necessary to decode sums of messages since the joint decoder is highly efficient and thus all messages could be directly decoded from each slot. However, our simulation results show that for a range of SNR and collision sizes of practical interest, decoding sums of messages does increase significantly the number of linearly independent combinations decoded in a slot. This is shown in Fig. 4, where the average number of innovative packets decoded in a slot plotted against the average SNR for the proposed seek and decode method ( $S \& D$ in the figure) and for a plain joint decoding scheme (JD in the figure), in which only single messages are decoded. For these simulations, we assumed block Rayleigh fading where the fading coefficients are constant during a packet transmission. A packet is said to be innovative if it can not be obtained as a linear combination in $G F(2)$ of previously decoded packets. Different curves for different collision sizes $k$ are shown. It can be seen how for larger $k$ and mid-low SNR values the gain of S\&D with respect to JD is significant (more than $25 \%$ gain at about $11 \mathrm{~dB}$ for $k=6$ ). The gain derives from the fact that, in a non negligible number of cases, it is possible do decode correctly the sum of two or more messages from a collision even if the single messages can not be decoded with a joint decoder. Note also that, once the decoding at the physical layer is finished, the receiver is left with $S$ sets of equations. Each of these sets derives from the decoding at the physical layer of each slot. Note also that, even if some (or all) of the sets of equations have not a full rank associated matrix, (i.e., not all of the colliding signals within a block can be decoded), it may still be possible to recover all the messages transmitted in a frame by applying matrix manipulations over the equivalent associated matrix in $G F\left(2^{n}\right)$.

\section{Conclusions}

We proposed a new random multiple access scheme for symbol-synchronous slotted aloha random access systems.

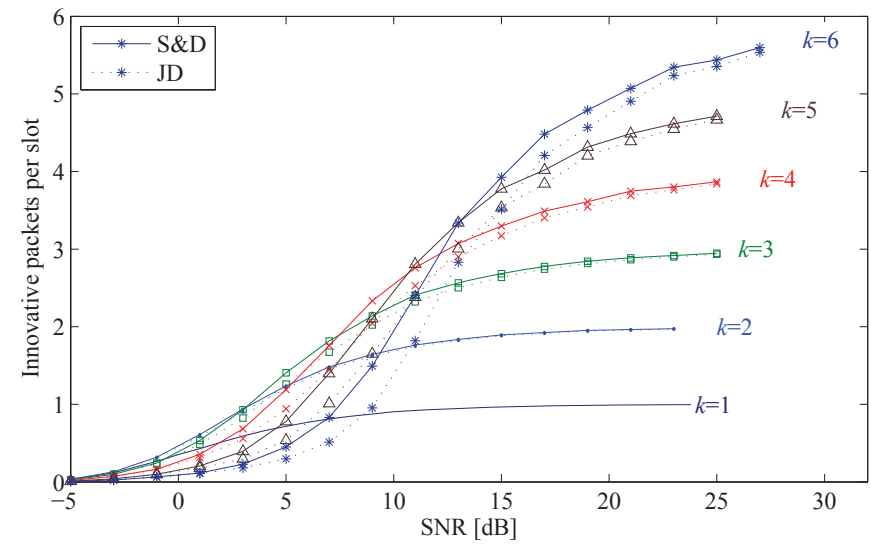

Fig. 4. Average number of innovative packets decoded in a slot plotted against the average SNR for the proposed seek and decode, S\&D, and for joint decoding, JD. Different curves for different collision sizes $k$ are shown. It can be seen how for larger $k$ and mid-low SNR values the gain of S\&D with respect to JD is significant (gain of around $20 \%$ at $10 \mathrm{~dB}$ for $k=4$ ).

Each node transmits several replicas of the same message within a frame after a pre-multiplication by a (pseudo)randomly chosen coefficient in $G F\left(2^{n}\right)$. The receiver tries to decode any many linear combination in $G F(2)$ of signals colliding in each slot as possible and then tries to recover all the messages transmitted within a frame treating the linear combinations decoded in the whole frame as a single system of equations in $G F\left(2^{n}\right)$. We presented analytical results for the throughput at system level and simulation results for the decoding process at the physical level. As future work we plan to optimize the multiple access scheme taking into account the decoder performance, which is a function of the collision size and the specific linear combination within a collision, with the aim of maximizing the system throughput and minimizing the packet error rate. At the physical level we plan to consider higher order modulations and different decoding approaches.

\section{REFERENCES}

[1] L.G. Roberts, "ALOHA packet system with and without slots and capture," SIGCOMM Computer Comm. Review, vol. 5, no. 2, pp. 28-42, Apr. 1975.

[2] S. Verdu, Multi User Detection, Cambridge University Press, 1998.

[3] J. G. Andrews, "Interference cancellation for cellular systems: a contemporary overview," IEEE Wireless Communications, vol. 12, no. 2, pp. 19-29, 2005.

[4] S. Ghez, S. Verdú, and S.C. Schwartz, "Stability properties of slotted ALOHA with multipacket reception capability," IEEE Trans. on Automatic Control, vol. 33, no. 7, pp. 640-649, July 1988.

[5] G. Choudhury and S. Rappaport, "Diversity ALOHA - A random access scheme for satellite communications," IEEE Trans. on Comm., vol. 31, no. 3, pp. 450-457, Mar. 1983. 
[6] E. Casini, R. De Gaudenzi, and O. del Rio Herrero, "Contention resolution diversity slotted ALOHA (CRDSA): An enhanced random access scheme for satellite access packet networks," IEEE Trans. on Wireless Comm., vol. 6, no. 4, pp. 1408-1419, Apr. 2007.

[7] G. Liva, "Graph-based analysis and optimization of contention resolution diversity slotted ALOHA," IEEE Trans. on Comm., vol. 59, no. 2, pp. 477-487, Feb. 2011.

[8] R. De Gaudenzi and O. del Rio Herrero, "Advances in random access protocols for satellite networks," in Int'l Workshop on Satellite and Space Comm., Siena, Italy, Sep. 2009.

[9] B. Nazer and M. Gastpar, "Computation over multiple-access channels," IEEE Trans. on Info. Theory, vol. 53, no. 10, pp. 3498-3516, Oct. 2007.

[10] B. Nazer and M. Gastpar, "Reliable physical layer network coding," Proceedings of the IEEE, vol. 99, no. 3, pp. 438-460, Mar. 2011.

[11] P. Popovski and H. Yomo, "The anti-packets can increase the achievable throughput of a wireless multi-hop network," in IEEE Int'l Conf. on Comm., Istanbul, Turkey, Dec. 2006.

[12] D. Wubben and Y. Lang, "Generalized sum-product algorithm for joint channel decoding and physical-layer network coding in twoway relay systems," in IEEE Global Telecommunications Conference (GLOBECOM), Miami, FL, U.S.A., 2010.

[13] S. Pfletschinger, "A practical physical-layer network coding scheme for the uplink of the two-way relay channel," in Asilomar Conference on Signals, Systems and Computers (ASILOMAR), Pacific Grove, CA, U.S.A., Nov. 2011.

[14] G. Cocco, C. Ibars, D. Gündüz, and O. del Rio Herrero, "Collision resolution in slotted ALOHA with multi-user physical-layer network coding," in IEEE Vehicular Technology Conf., Budapest, Hungary, May 2011

[15] G. Cocco and C. Ibars, "On the feasibility of satellite M2M systems," in AIAA International Communications Satellite Systems Conference (ICSSC), Ottawa, Canada, Sep. 2012.

[16] G. Cocco, N. Alagha, C. Ibars, and S. Cioni, "Network-coded diversity protocol for collision recovery in slotted-aloha networks," Wiley's Int'l Journal of Satellite Comm. and Networking, November 2013.

[17] G. Cocco, N. Alagha, C. Ibars, and S. Cioni, "Practical issues in multiuser physical layer network coding," in IEEE Advanced Satellite Mobile Systems Conf., Baiona, Spain, Sep. 2012.

[18] S. Pfletschinger, "Joint decoding of multiple non-binary LDPC codewords," in IEEE ICC Workshop on Massive Uncoordinated Access Protocols (MASSAP), Sydney, Australia, 2014, submitted.

[19] The Consultative Committee for Space Data Systems (CCSDS), TM Synchronization and Channel Coding, August 2011, CCSDS 131.0-B2 . 